\title{
Corrected Bohr-Sommerfeld Quantum Conditions for Nonseparable Systems*
}

\author{
JOSEPH B. KELLER \\ Institute of Mathematical Sciences, New York University, New York, New York
}

For a separable or nonseparable system an approximate solution of the Schrödinger equation is constructed of the form $A e^{i \hbar^{-1} S}$. From the singlevaluedness of the solution, assuming that $A$ is single-valued, a condition on $S$ is obtained from which follows $A$. Einstein's generalized form of the Bohr-Sommerfeld-Wilson quantum conditions. This derivation, essentially due to $\mathrm{L}$. Brillouin, yields only integer quantum numbers. We extend the considerations to multiple valued functions $A$ and to approximate solutions of the form

$$
\sum A_{k} \exp \left(i \hbar^{-1} S_{k}\right)
$$

In this way we deduce the corrected form of the quantum conditions with the appropriate integer, half-integer or other quantum number (generally a quarter integer). Our result yields a classical mechanical principle for determining the type of quantum number to be used in any particular instance. This fills a gap in the formulation of the "quantum theory", since the only other method for deciding upon the type of quantum number-that of Kramers-applies only to separable systems, whereas the present result also applies to nonseparable systems.

In addition to yielding this result, the approximate solution of the Schrödinger equation-which can be constructed by classical mechanics-may itself prove to be useful.

\section{INTRODUCTION}

In the "quantum theory" the motion of a system is described by classical mechanics but certain constants of the motion are restricted to be integers. These restrictions are called the quantum conditions and the integers occurring in them are called the quantum numbers. In many cases better agreement between theory and observation is obtained if half-integer quantum numbers are employed instead of integers. However no theoretical principle is available to determine whether an integer, half-integer or other quantum number is to be used in any particular case. It is the purpose of this article to provide such a principle.

* This article is based upon a report (1) sponsored by the Geophysics Research Directorate, Air Force Cambridge Research Center, Air Research and Development Command, under Contract No. AF19(122)-463. 
Historically the problem of deciding between integer and half-integer quantum numbers was circumvented by the invention of quantum mechanics, which replaced the "quantum theory". Therefore the discovery of a procedure for deciding between the two kinds of quantum numbers might now be considered to be of purely academic interest. However this is not necessarily the case because the procedure can still be used in the approximate solution of quantum mechanical problems.

1 way of deciding between integer and half-integer quantum numbers for reparable systems was found by Kramers (2) hy means of an approximate solution of the Schrödinger equation. His result also showed that for such systems these conditions are consequences of quantum mechanies in the limit as Planck's coustant $h$ tends to zero. Another derivation of the quantum conditions from the Schrödinger equation was given by Brillouin (3). It was more gener:ul than Kramers' since it applied to both separable and nonseparable systems, but it was incorrect since it yielded only integer quantum numbers. Brillonin: argument is essentially the following:

Consider a quantum mechanical system with $X$ roordinate operators $4 r, 1$ conjugate momentum operators $p_{r} \equiv-i \hbar^{-1}\left(\partial ; q_{r}\right), r=1, \cdots, N$, and IIamiltonian operator $H\left(q_{r}, p_{r}, t\right)$. Let the Schrödinger representor of the state af the system be $\Psi\left(q_{r}, t\right)$. Suppose that $\Psi$ is approximately equal to $\Psi_{11}$ defined hy

$$
\Psi_{11}=A\left(q_{r}, t, \hbar\right) \exp \left[i \hbar^{-1} S\left(q_{r}, t, \hbar\right)\right] \text {. }
$$

The function $\Psi$ and hence $\Psi_{0}$ must be single valued. Therefore if $s$ is multiple ralued and if $\Delta S$ denotes the difference between any two of its ralues, it is neressitry that

$$
\Delta S=n h \text {. }
$$

Here $n$ is an integer. This condition guarantees that $\Psi_{0}$ will be single valued eren if $S$ is not. The quantum conditions with integer quantum numbers follow from (2), as will be shown below. We will also show that (2) is equivalent to the quantum condition postulated by Einstein (4) in generalizing the BohrSommerfeld-Wilson conditions to nonseparable systems.

We note that Brillouin's argument assumes that $A$ is single ralued. Howerer if $A$ is not single valued then in order for $\Psi_{0}$ to be single valued (2) must he replaced by

$$
\Delta S=h\left[n+i \frac{\Delta \log A}{2 \pi}\right] .
$$

Suppose, for example, that two values of $A$ differ only in sign. Then $\Delta \log A=$ $-i \pi$ and (3) yields

$$
\Delta S=h\left[n+\frac{1}{2}\right] .
$$

From (4) the quantum condition with a half-integer quantum number follows. 
We have just indicated how to modify Brillouin's argument in order to obtain the appropriate integer, half-integer or other quantum number. To complete the argument we must examine the amplitude $A$. This will be done in the next sections. However we must first make another change in the method. Instead of (1), we will assume that $\Psi_{0}$ is a sum of terms given by

$$
\Psi_{0}=\sum_{k=1}^{M} A_{k}\left(q_{r}, t, \hbar\right) \exp \left[i \hbar^{-1} S_{k}\left(q_{r}, t, \hbar\right)\right] .
$$

This more general form of approximate solution is requircd in almost all problems in which $S$ is multiple valued. The various functions $S_{k}$ and $A_{k}$ will be considered to be different branches of multiple valued functions $S$ and $A$. We will also make our considerations more definite by assuming that $\Psi$ is asymptotic to $\Psi_{0}$ as Planck's constant $\hbar$ tends to zero. This viewpoint was introduced by Birkhoff (5).

\section{ASYMPTOTIC SOLUTION OF THE SCHRÖDINGER EQUATION}

The function $\Psi$ satisfies the Schrödinger equation

$$
H\left(q_{r}, p_{r}, t\right) \Psi\left(q_{r}, t\right)=i \hbar \frac{\partial \Psi}{\partial t}\left(q_{r}, t\right) .
$$

Upon inserting the expression (5) for $\Psi_{0}$ into (6) and considering the leading terms in $\hbar$ we obtain equations for the $A_{k}$ and $S_{k}$. These equations were derived by Dirac $(6)$ when $\Psi_{0}$ consists of a single term and the same analysis applies when $\Psi_{0}$ is a sum of terms. The result is that each $S_{k}$ satisfies the classical Hamilton-Jacobi equation

$$
H\left(q_{r}, \frac{\partial S_{k}}{\partial q_{r}}, t\right)=-\frac{\partial S_{k}}{\partial t} .
$$

The equation for each $A_{k}$, written in terms of $P_{k}=A_{k}{ }^{2}$ and

$$
v_{r}=\frac{\partial H}{\partial p_{r}}\left(q_{r}, \partial S / \partial q_{r}, t\right),
$$

is

$$
\frac{\partial P_{k}}{\partial t}+\sum_{r=1}^{N} \frac{\partial}{\partial q_{r}}\left[v_{r} P_{k}\right]=0 .
$$

Equation (8) is the Liouville equation of classical statistical mechanics for the probability distribution $P_{k}$ of a classical mechanical system with Hamiltonian $H$. It is a special form because $P_{k}$ depends only upon $q_{r}$ and $t$, but not upon $v_{r}$ or $p_{r}$ as is usual in classical mechanics. This is a consequence of the fact that quantum mechanics does not yield joint probability distributions of conjugate 
variables. It is to be noted that $P_{k}$ is not necessarily positive, nor even real, in the present case. We will also permit $S_{k}$ to be complex.

Let us solve (7) when $H$ is independent of $t$. Then $H=E$, where the constant $E$ is the total energy of the system. Now if $q_{r}(t), p_{r}(t)$ denote a trajectory, then the function $S_{k}$ is given at any point of the trajectory in terms of its ralue $S_{k}(0)$ at some fixed point on the trajectory by

$$
\begin{aligned}
S_{k}\left(q_{r}, t, \hbar\right) & =S_{k}(0)+\int_{0}^{t} \sum_{r=1}^{N} p_{r}(\tau) \frac{d q_{r}(\tau)}{d \tau} d \tau-E t \\
& =S_{k}(0)+\int_{0}^{t} \sum_{r=1}^{N} p_{r} d q_{r}-E t
\end{aligned}
$$

Equation (8) can also be solved at once if $P_{k}$ is independent of $t$, for then it hecomes

$$
\sum_{r=1}^{N} \frac{\partial}{\partial q_{r}}\left[v_{r} P_{k}\right]=0 .
$$

Fquation (10) asserts that the probability flux is divergenceless, and therefore by applying Gauss' theorem to a tube of trajectories we obtain

$$
P_{k} v=P_{k}(0) v_{0} \frac{d \sigma_{0}}{d \sigma}
$$

In $(11)$

$$
v=\left[\sum_{r=1}^{N} v_{r}^{2}\right]^{1 / 2}
$$

and $d \sigma$ is the normal cross sectional area of the tube of trajectories, both evaluated at the same point at which $P_{k}$ is cvaluated. The corresponding quantities $\gamma_{0}$, $d \sigma_{0}$, and $P_{k}(0)$ are evaluated at some other point on the same trajectory, and thus (11) merely asserts the conservation of "probability". Actually (1I) holds in the limit as $d \sigma_{0}$ tends to zero, in which case $d \sigma / d \sigma_{0}$ denotes the Jacobian of the mapping of one cross section onto another by means of trajectories. The problem of Rutherford scattering has been treated by Gordon ( $\gamma$ ) and by the present author (1) by means of Eqs. (9) and (11).

\section{THE QUANTUM CONDITIONS}

Quantum mechanics requires that $\Psi$ be a single valued function of the $q_{r}$ and therefore $\Psi_{0}$ must satisfy the same condition. Consequently each term in the expression (5) for $\Psi_{0}$ must be single ralued. As we have shown in the Introdurtion, this requires that each $S_{k}$ and the corresponding $A_{k}$ must satisfy the condition (3). If any $A_{k}$ is single valued, this condition becomes the simpler condition (2) for the corresponding $S_{k}$. 
The differences $\Delta S_{k}\left(q_{r}, t\right)$ and $\Delta \log A_{k}\left(q_{r}, t\right)$ are expressible as line integrals over some closed curve in $q_{r}$ space beginning and ending at $q_{r}$. In terms of these integrals, (3) becomes, for each value of $k$,

$$
\oint \nabla S \cdot d s=h\left[n+\frac{i}{2 \pi} \oint \nabla \log A \cdot d s\right] .
$$

Equation (12) must hold for every closed curve in the $q_{r}$ space, since only then will $\Psi_{0}$ be single valued at every point. However the line integrals in (12) have the same value for every two closed curves which are deformable into one another without crossing a singularity of the integrand. For example, they are zero for a curve which can be deformed into a point. There are, in general, only a finite number of classes of independent curves which cannot be deformed into points. Any other curve is deformable into a linear combination of such curves with integer coefficients. Therefore (12) will be satisfied for all curves if it is satisfied by one curve in each of the independent classes of curves. Thus we have, in general, a finite number of quantum conditions, in each of which the integer $n$ is arbitrary.

Since $\nabla S \cdot d s=\sum_{r} p_{r} d q_{r},(12)$ can be rewritten as

$$
\oint \sum_{r} p_{r} d q_{r}=h\left[n+\frac{i}{2 \pi} \oint \nabla \log A \cdot d s\right] \text {. }
$$

When $A_{k}$ is single valued, the conditions (13) become exactly the quantum conditions postulated by Einstein (4) for a system in a steady state of constant energy. He pointed out that these quantum conditions are invariant under a contact transformation of variables because $\sum p_{r} d q_{r}$ is invariant. If the variables are separable in the Hamilton-Jacobi equation, so that each $p_{r}$ can be expressed in terms of the corresponding $q_{r}$ alone, and if $A_{k}$ is single valued, then these conditions reduce to the well known Bohr-Sommerfeld-Wilson quantum conditions for a separable system in a steady state of constant energy.

In order to clarify the conditions (12) and (13) let us consider a multiple valued solution $S$ of the Hamilton-Jacobi equation. Such a solution generally has infinite multiplicity, i.e., an infinite number of different values or branches. However only a finite number of its branches, say $M$ of them, are essentially distinct. Every other branch differs from one of these branches by an additive constant. Therefore the function $\nabla S$ will have only the finite multiplicity $M$ since any two branches differing by a constant yield the same value for $\nabla S$. Let us introduce an $M$-sheeted $q_{r}$ space and associate one branch of the function $\nabla S$ with each sheet. We will denote each sheet by an integer $k$ and the corresponding branch of $\nabla S$ by $\nabla S_{k}$, with $k$ ranging from one to $M$. Any two sheets-say sheets $j$ and $k$-are to be joined together at all points where $\nabla S_{j}=\nabla S_{k}$. Furthermore if $\nabla S$ is defined in only part of the $q_{r}$ space then only that part is covered 
by additional sheets. The $M$ sheeted $q_{r}$ space so constructed is called the covering space for the function $\nabla S$. Its main property is that on it $\nabla S$ is a single valued function. The Riemann surfaces of function theory are examples of such spaces.

The same considerations may be applied to the multiple valued function $\log A$. Its gradient has the same multiplicity $M$ as does $\Gamma S$ as we see from (8) and its different branches become equal where those of $\nabla S$ do. Therefore the sime covering space on which $\nabla S$ is single valued also serves as the covering space for $\nabla \log A$. Consequently the line integrals in (12) and (13) may be thought of as being evaluated along a closed path on this covering space. Then the omision of the subscript $k$ in (12) and (13) is appropriate since any number of branches may be involved in each integral. Also the question of whether one closed curve is deformable into another becomes clear in this space. Furthermore, the independent closed curves can be recognized as the basis of the fundamental group of the covering space. In this way we see that the topology of the covering spares determines the number of quantum conditions. This number is just the number of closed curves in the basis of the fundamental group.

Let us now consider the evaluation of

$$
\Delta \log A=\mathscr{f} \nabla \log A \cdot d s .
$$

We will restrict our attention to steady states since then $d^{2}=P$ is explicitly given by (11). From this equation we see that $P$ becomes infinite whenever $u d \sigma$ becomes zero. We will call points at which this oceurs caustic points, in analogy with optics where points at which $d \sigma=0$ are so named. A locus of caustic points. is called a caustic of the family of trajectories associated with the $S$ function under consideration. Those caustics which correspond to the vanishing of $d \sigma$ are envelopes of the family of trajectories. Therefore $\mathrm{TS}$ is multiple valued near these surfaces. Conseguently such causties are the loci of points at which two different branches of $\nabla S$ become equal. Thus these caustics form the boundaries at which different shects of the covering space for $\tau S$ are joined together. Those mustic's at which $v=0$ also form part of these boundaries, assuming that each $p_{r}$ either changes sign along each trajectory on which $r$ vanishes or is identically zero near the caustic. l'or then $\nabla S$, which has the $p_{\text {r an }}$ components, reverses its direction at the caustir. Thus this type of caustic is also a boundary on which two hranches of $\nabla S$ join.

We have seen that $A$ becomes infinite on a caustic and that al caustic must be crossed by a path which goes from one sheet of the covering space to another. It is well known in optics that the phase of $A$ is retarded by $\pi / 2$ (i.e., $A$ is multiplied by $e^{-i \pi / 2}$ ) on a ray which passes through a caustic on which $d \sigma$ vanishes simply. (The positive direction along a ray is the direction of $\Gamma S$.) Furthermore the phase is retarded by $\pi$ on a ray passing through a focus, which is a caustic point at which $d \sigma$ vanishes to the second order. The usual method for proving these farts.s 
is based upon the asymptotic evaluation of certain double integrals which represent the wave function. These integral representations are deduced from Green's theorem, and the integrals are evaluated by the method of stationary phase. Both of these considerations can be immediately extended to problems such as the present one in which the number of dimensions is $N$. The result is this: The phase of $A$ is retarded by $m \pi / 2$ on a trajectory which passes through a caustic on which $d \sigma$ vanishes to the $m$ th order. We may replace the statement " $d \sigma$ vanishes to the $m$ th order" by the equivalent statement "the dimensionality of the cross section of a tube of trajectories is reduced by $m$ ". This result is an analogue for partial differential equations of the Kramers connection formulas which are employed in the WKB treatment of ordinary differential equations. In the present case the caustics play the role of the turning points.

From the foregoing analysis we see that $\log A$ changes by $-i m \pi / 2$ along a path which passes from one sheet of the covering space to another in the direction - of $\nabla S$. Here the positive integer $m$ is the number of dimensions "lost" by a tube of trajectories at the caustic. Obviously $m$ must be replaced by $-m$ if the path is traversed in the opposite direction. Considerations similar to those outlined above show that at the caustics on which $v$ vanishes the phase of $A$ is also retarded by $m \pi / 2$, so $\log A$ changes by $-i m \pi / 2$ where $m$ is the number of $p_{r}$ which change sign at the caustic.

The total change $\Delta \log A$ along a closed curve is generally just the change associated with the various caustics through which the curve passes. Therefore, in general, we have

$$
\frac{i}{2 \pi} \Delta \log A=\frac{i}{2 \pi} \oiint \nabla \log A \cdot d s=\frac{m}{4} .
$$

Here $m$ denotes the total number of dimensions "lost" by the trajectories at the caustics through which the curve passes plus the number of $p_{r}$ which change sign at the $v=0$ caustics through which the curve passes. In evaluating $m$ account must be taken of whether the curve traverses the caustic in the direction of increasing or decreasing $S$. When (14) is used the quantum conditions (13) finally become

$$
\mathscr{S} \sum p_{r} d q_{r}=h\left[n+\frac{m}{4}\right] .
$$

These are the corrected quantum conditions for separable or nonseparable systems. In each quantum condition the positive integer $n$ is arbitrary but the integer $m$ is determined by the considerations described above.

THE "CLASSICAL $\Psi$ FUNCTION"

We will call the function $\Psi_{0}$ given by (5) the "classical $\Psi$ function" because it can be constructed by classical mechanical considerations alone. In spite of this 
the probabilities computed from $\left|\Psi_{0}\right|^{2}$ still show quantum mechanical interference effects if the sum in (5) contains more than one term.

If only one term occurs in (5) then $\left|\Psi_{0}\right|^{2}=\left|A^{2}\right|^{2} \exp \left(-2 h^{-1} I m S\right)$, and the probability, as well as $\Psi_{0}$ itself, is exponentially damped in regions where $\operatorname{ImS}>0$. The probability in such regions vanishes as $h$ tends to zero, corresponding to the fact that these regions are excluded in classical mechanics. This can be seen from the fact that the solution of the Hamilton-Jacobi equation is not real there. The exponential tail shows that $\Psi_{0}$ describes such quantum mechanical effects as "tunneling".

If $\operatorname{ImS}=0$ and $|A|^{2}=A^{2}=P$, then $\left|\Psi_{0}\right|^{2}=P$. Thus as $h$ tends to zero the quantum mechanical probability distribution approaches that given by the Liouville equation of classical statistical mechanics, and we may say that quantum mechanies approaches classical statistical mechanics, as $h$ tends to zero. The customary statement that quantum mechanics approaches classical mechanics is thus not strictly correct, but holds only when the initial data are such that $P=0$ except on one trajectory, in which case classical statistical mechanics reduces to classical mechanics. Since the classical method of computing differential scattering cross sections is actually based on classical statistical mechanic's, the preceding considerations show that the quantum mechanical cross sections will approach them as $h$ tends to zero when only one term occurs in (5).

Finally it is to be noted from (13) or (15) that $h$ and $n$ enter the solution only in the combination $\left(n+\frac{1}{1} m\right) h$. In some problems the solution for fixed $n$ does not have the asymptotic behavior assumed in the derivation. However when the limit in which $n$ becomes infinite while $h$ becomes zero and $\left(n+1_{4} m\right) h$ is constant is considered, the assumed asymptotic behavior may result. In such cases the asymptotic solution applies only for high quantum numbers $n$.

\section{AN EXAMPLE-THE HARMONIC OSCILLATOR}

To exemplify the preceding results, let us consider the steatdy state of an onedimensional harmonic oscillator of mass $m$, energy $E$, frequency $\nu_{0}$, and momentum $p$. Recalling that $S_{x}=p$, we have from the definition of momentum

$$
S_{x}=p= \pm\left(2 m E-m \nu_{0} x^{2}\right)^{1 \cdot 2} .
$$

We see that $\nabla S=S_{x}$ is real and double valued in the interval $-x_{0} \leqq x \leqq x_{0}$ where $x_{0}=\left(2 E \nu_{0}^{-1}\right)^{1 / 2}$. The two branches of $S_{x}$ become equal at the endpoints of this interval. Thus the covering space for $S_{x}$ consists of two line segments joined together at their two ends. This space is topologically equivalent to a circle, and there is only one basic closed curve on it. Therefore there is only one quantum condition. The closed curve passes through the two caustics $x= \pm x_{0}$ at both of which $v=m^{-1} p$ vanishes and $p$ changes sign. Thus in (15) we have $m=2$ so (15) becomes 


$$
\int_{-x_{0}}^{x_{0}}\left(2 m E-m \nu_{0} x^{2}\right)^{1 / 2} d x-\int_{x_{0}}^{-x_{0}}\left(2 m E-m \nu_{0} x^{2}\right)^{1 / 2} d x=h\left(n+\frac{1}{2}\right) .
$$

Equation (17) is just the result given by the usual WKB method in this case. A similar analysis holds for a particle in any one dimensional potential well.

To construct $\Psi_{0}$ we note that $d \sigma_{0} / d \sigma=1$ in the present case so (11) yields, with $A_{0}$ a constant,

$$
A=A_{0} v^{-1 / 2} \text {. }
$$

Inserting $v=m^{-1} p$ in (18) with $p$ given by (16) we obtain the two results

$$
\begin{gathered}
A_{+}=A_{0} m^{1 / 4}\left(2 E-\nu_{0} x^{2}\right)^{-1 / 4} \\
A_{-}=e^{-i \pi / 2} A_{0} m^{3 / 4}\left(2 E-\nu_{0} x^{2}\right)^{-1 / 4} .
\end{gathered}
$$

The phase retardation represented by the factor $e^{-i \pi / 2}$ in (20) accounts for the phase shift which occurs upon passing through either caustic. In the present example it arises formally when $p$ is negative and the square root of $p^{-1}$ is taken. Of course our previous considerations are necessary to ensure that we take the correct root.

Upon inserting (19), (20), and (16) into (5) and setting $S\left(x_{0}\right)=0$, we obtain for $\Psi_{0}(x)$ in the region $|x| \leqq x_{0}$,

$$
\begin{aligned}
\Psi_{0}(x)= & A_{0} m^{1 / 4}\left(2 E-\nu_{0} x^{2}\right)^{-1 / 4}\left\{\exp \left[-i \hbar^{-1} \int_{x}^{x_{0}}\left(2 m E-m \nu_{0} x^{2}\right)^{1 / 2} d x\right]\right. \\
& \left.+\exp \left[-i \frac{\pi}{2}+i \hbar^{-1} \int_{x}^{x_{0}}\left(2 m E-m \nu_{0} x^{2}\right)^{1 / 2} d x\right]\right\} \\
= & e^{-i(\pi / 4)} A_{0} m^{1 / 4}\left(2 E-\nu_{0} x^{2}\right)^{-1 / 4} \cos \left[\hbar^{-1} \int_{x}^{x_{0}}\left(2 m E-m \nu_{0} x^{2}\right)^{1 / 2} d x-\frac{\pi}{4}\right] .
\end{aligned}
$$

This is the usual WKB result. We may obtain the result for a particle in any one dimensional potential $V(x)$ by replacing $\nu_{0} x^{2}$ by $2 V(x)$ in $(21)$.

RECEIVED: January 23, 1958

\section{REFERENCES}

1. J. B. Keller, New York University, Mathematics Research Group, Research Report No. CX-10, July, 1953 (unpublished).

2. H. A. Kramers, $Z$. Physile 39, 828-840 (1926).

3. L. BRillouin, J. phys. radium 7, 353-368 (1926).

4. A. Einsteis, Verhandl. deut. physik. Ges. (1917). References to prior work are given in this paper.

5. G. D. Birkhoff, Amer. Math. Soc. Bull. 39, 681-700 (1933).

6. P. A. M. Dirac, "The Principles of Quantum Mechanics," 3rd ed., pp. 121-123. Oxford Univ. Press, London and New York, 1947.

7. W. Gondon, Z. Physik 48, 180 (1928). 